International Mathematical Forum, 2, 2007, no. 50, 2481 - 2490

\title{
Resolvent Positive Operator and Two-Parameter Abstract Cauchy Problem
}

\author{
M. Khanehgir ${ }^{1}$ and R. Ghorbanpoor \\ Department of Mathematics \\ Islamic Azad University of Mashhad \\ Mashhad P.O. Box 413-91735, Iran
}

\begin{abstract}
We use the semigroup theory to study the homogeneous two-parameter abstract Cauchy problem 2-RACP

$$
\left\{\begin{array}{l}
\frac{\partial}{\partial t_{i}} u\left(t_{1}, t_{2}\right)=H_{i} u\left(t_{1}, t_{2}\right) \\
i=1,2 \quad t_{i} \in\left[0, a_{i}\right] \\
u(0,0)=u_{0}, \quad u_{0} \in E .
\end{array}\right.
$$

Where $u$ is a function from $\left[0, a_{1}\right] \times\left[0, a_{2}\right]$ to ordered Banach space $E$ whose positive cone is normal and generating and $a_{1}, a_{2}>0, H_{i}$ : $D\left(H_{i}\right) \subseteq E \rightarrow E, i=1,2$, is a resolvent positive operator. We discuss the existence and uniqueness of solution of 2-RACP. In fact, we claim that if $\left(H_{1}, H_{2}\right)$ is the generator of a two-parameter integrated semigroup $\left\{S\left(t_{1}, t_{2}\right)\right\}_{t_{1}, t_{2} \geq 0}$ and $u_{0} \in \cap_{i=1}^{2} D\left(H_{i}^{2}\right)$ then 2-RACP has a unique solution.
\end{abstract}

Mathematics Subject Classification: 34A12, 34A30, 47D03, 47D62, 47D99, 35D05

Keywords: N-parameter semigroup, two-parameter resolvent positive operator, two-parameter integrated semigroup and two-parameter abstract Cauchy problem

\section{Introduction}

Let $X$ be a Banach space, $B(X)$ is the Banach space of all bounded linear operators on $X$ and $\mathbb{R}_{+}^{n}=\left\{\left(t_{1}, t_{2}, \ldots, t_{n}\right): t_{i} \geq 0, i=1,2, \ldots, n\right\}$.

By an n-parameter semigroup of operators we mean a homomorphism $W$ : $\left(\mathbb{R}_{+}^{n},+\right) \rightarrow B(X)$ for which $W(0)=I$ and denote it by $\left(X, \mathbb{R}_{+}^{n}, W\right)$. Let

\footnotetext{
${ }^{1}$ khanehgir@mshdiau.ac.ir
} 
now $\left\{e_{i}\right\}_{i=1}^{n}$ is the standard basis of $\mathbb{R}^{n}$. Trivially for $s \in \mathbb{R}^{+}$, the component $u_{i}(s)=W\left(s e_{i}\right)$ of $W$ defines a one-parameter semigroup of operators, $i=1,2, \ldots, n$.

Also for each integers $0 \leq i, j \leq n$, the n-parameter semigroup property implies that, $u_{i}(s) u_{j}\left(s^{\prime}\right)=u_{j}\left(s^{\prime}\right) u_{i}(s)$. The n-parameter semigroup $\left(X, \mathbb{R}_{+}^{n}, W\right)$ is called strongly (respectively, uniformly) continuous if for each $i=1,2, \ldots, n$, the one-parameter components $u_{i}(s)=W\left(s e_{i}\right)$ are strongly (respectively, uniformly) continuous. One can prove that the n-parameter semigroup $\left(X, \mathbb{R}_{+}^{n}, W\right)$ is strongly continuous if and only if $\lim _{t \in \mathbb{R}_{+}^{n}, t \rightarrow 0} W(t) x=x$, for all $x \in X$, and it is uniformly continuous if and only if $\lim _{t \in \mathbb{R}_{+}^{n}, t \rightarrow 0} W(t)=I$. Consider a n-parameter semigroup of operators $\left(X, \mathbb{R}_{+}^{n}, W\right)$ and let $H_{i}, i=1,2, \ldots, n$, be the infinitesimal generator of the component semigroup $\left\{u_{i}(t)\right\}_{t \geq 0}$ of $W$, $i=1,2, \ldots, n$. We shall think of $\left(H_{1}, H_{2}, \ldots, H_{n}\right)$ as the infinitesimal generator of $\left(X, \mathbb{R}_{+}^{n}, W\right)$.

N-parameter semigroups of operators introduced by Hille in 1944 and one can see some of their properties in [5], [6] and [7].

If $\mathrm{W}$ is a $C_{0}$-n-parameter semigroup of operators then by the Hille-Yosida theorem, $H_{i}, i=1,2, \ldots, n$, is a closed and densely defined operator.

In [3] one can see that if $\left(X, \mathbb{R}_{+}^{n}, W\right)$ is a $C_{0}$-n-parameter semigroup of operators with the infinitesimal generator $\left(H_{1}, H_{2}, \ldots, H_{n}\right)$ and $D\left(H_{i}\right) \subseteq X$ be the domain of $H_{i}, i=1,2, \ldots, n$, then

(a) There is $M \geq 1$ and $\omega_{i} \in \mathbb{R}, i=1,2, \ldots, n$, such that $\left\|W\left(t_{1}, t_{2}, \ldots, t_{n}\right)\right\| \leq$ $M e^{\sum_{i=1}^{n} t_{i} \omega_{i}}$. So $\left\|W\left(t_{1}, \ldots, t_{n}\right)\right\|$ is bounded in any compact subset of $\mathbb{R}_{+}^{n}$.

(b) If $x \in D\left(H_{i}\right)$, so does $W(t) x$, for each $t \in \mathbb{R}_{+}^{n}$, and $H_{i} W(t) x=W(t) H_{i} x$, $i=1,2, \ldots, n$.

Also we have the Hille-Yosida theorem for n-parameter semigroups as follows $([6])$

$\left(H_{1}, \ldots, H_{n}\right)$ is the infinitesimal generator of a $C_{0}$-n-parameter semigroup $\{W(t)\}_{t \in \mathbb{R}_{+}^{n}}$, satisfying $\left\|W\left(t_{1}, \ldots, t_{n}\right)\right\| \leq M_{0} e^{\sum_{i=1}^{n} t_{i} \omega_{i}}$, for some $M_{0} \geq 1$ and $\omega_{i}>0, i=1, \ldots, n$, if and only if

(a) $H_{i}$ is a closed densely defined operator, $i=1, \ldots, n$, and $R\left(\lambda^{\prime}, H_{j}\right) R\left(\lambda, H_{i}\right)=$ $R\left(\lambda, H_{i}\right) R\left(\lambda^{\prime}, H_{j}\right)$, for all $\lambda>\omega_{i}$ and $\lambda^{\prime}>\omega_{j}$ and integers $1 \leq i, j \leq n$.

(b) For each $i=1, \ldots, n,\left[\omega_{i}, \infty\right) \subseteq \rho\left(H_{i}\right)$ (the resolvent set of $H_{i}$, see [9]) and there is $M \geq 1$, such that

$\left\|R\left(\lambda, H_{i}\right)^{k}\right\| \leq \frac{M}{\left(\operatorname{Re} \lambda-\omega_{i}\right)^{k}}, i=1, \ldots, n$, and $\operatorname{Re} \lambda>\omega_{i}$.

Now we define one-parameter integrated semigroup which is introduced by W. Arendt in 1986 (see [1], [2]).

Definition 1.1 Let $X$ be a Banach space. An integrated semigroup is a family $\left(S_{t}\right)_{t \geq 0}$ (sometimes we apply $\{S(t)\}_{t \geq 0}$ for this notion) of bounded linear operators $S_{t}$ on $X$ with the following properties:

(a) $S_{0}=0$. 
(b) $t \rightarrow S_{t}$ is strongly continuous.

(c) $S_{s} S_{t}=\int_{0}^{s}\left(S_{r+t}-S_{r}\right) d r$, for $s, t \geq 0$.

A short calculation shows that (c) can also be written as $S_{s} S_{t}=\int_{0}^{s+t} S_{r} d r-$ $\int_{0}^{s} S_{r} d r-\int_{0}^{t} S_{r} d r$, for all $s, t \geq 0$. A consequence of this is $S_{s} S_{t}=S_{t} S_{s}$, for all $s, t \geq 0$.

Definition 1.2 An integrated semigroup $\left(S_{t}\right)_{t \geq 0}$ is called exponentially bounded, if there exist constants $M \geq 0$ and $\omega \in \mathbb{R}$ such that $\left\|S_{t}\right\| \leq M e^{\omega t}$, for all $t \geq 0$. Moreover $\left(S_{t}\right)_{t \geq 0}$ is called non-degenerate if $S_{t} x=0$, for all $t \geq 0$ implies that $x=0$.

Let $\{S(t)\}_{t>0}$ be an exponentially bounded integrated semigroup i.e. \| $S(t) \| \leq M e^{\omega t}$, for some constants $M \geq 0$ and $\omega \in \mathbb{R}$, and for all $t \geq 0$. Define $R(\lambda)=\lambda \int_{0}^{\infty} e^{-\lambda t} S(t) d t$, for $\lambda>\omega$. Then $k e r R(\lambda)$ is independent of $\lambda>\omega$ (see [2] theorem 1.6.9). Hence $R(\lambda)$ is injective if and only if $\{S(t)\}_{t \geq 0}$ is non-degenerate. In this case there exists a unique operator A satisfying $(\omega, \infty) \subset \rho(A)$ such that $R(\lambda)=(\lambda-A)^{-1}$, for all $\lambda>\omega$. This operator is called the generator of $\{S(t)\}_{t \geq 0}$.

In the following theorem we characterize the generator of a one parameter integrated semigroup.

Theorem 1.3 Let $A$ be a densely defined operator such that $(a, \infty) \subset \rho(A)$ for some $a \geq 0$. Let $M \geq 0, \omega \in(-\infty, a]$, then the following assertions are equivalent

(i) A generates an integrated semigroup $\{S(t)\}_{t \geq 0}$ satisfying $\|S(t)\| \leq M e^{\omega t}$, for all $t \geq 0$.

(ii) $\|(\lambda-\omega)^{k+1}[R(\lambda, A) / \lambda]^{(k)} / k$ ! $\| \leq M$, for all $\lambda>a, k=0,1,2, \ldots$.

Proof. See [2].

We can extend the notion of one-parameter integrated semigroup to twoparameter integrated semigroup as follows. (see [8]).

Let $\{W(t, s)\}_{t, s \geq 0}$ be a $C_{0}$-two-parameter semigroup on a Banach space $X$. We define a family $\left\{S\left(t_{1}, s_{1}\right)\right\}_{t_{1}, s_{1} \geq 0}$ of bounded linear operators on $X$ as follows:

$$
S\left(t_{1}, s_{1}\right)=\int_{0}^{t_{1}} W(t, 0) d t+\int_{0}^{s_{1}} W(0, s) d s .
$$

The family $\left\{S\left(t_{1}, s_{1}\right)\right\}_{t_{1}, s_{1} \geq 0}$ have the following properties:

(a) $S(0,0)=0$.

(b) $\left(t_{1}, s_{1}\right) \rightarrow S\left(t_{1}, s_{1}\right)$ is strongly continuous.

(c) $S\left(t_{1}, s_{1}\right) S\left(t_{2}, s_{2}\right)=\int_{0}^{t_{1}} S\left(t+t_{2}, 0\right)-S(t, 0) d t+S\left(t_{1}, 0\right) S\left(0, s_{2}\right)$ 


$$
\begin{aligned}
& +S\left(0, s_{1}\right) S\left(t_{2}, 0\right)+\int_{0}^{s_{1}} S\left(0, s_{2}+s\right)-S(0, s) d s \\
& =\left[S\left(t_{1}, 0\right)+S\left(0, s_{1}\right)\right]\left[S\left(t_{2}, 0\right)+S\left(0, s_{2}\right)\right] .
\end{aligned}
$$

(d) $S\left(t_{1}, 0\right) S\left(0, s_{1}\right)=S\left(0, s_{1}\right) S\left(t_{1}, 0\right)$.

Definition 1.4 Let $X$ be a Banach space and consider the family $\{S(t, s)\}_{t, s \geq 0}$ of bounded linear operators on $X$. We say that $\{S(t, s)\}_{t, s \geq 0}$ is a two-parameter integrated semigroup of operators on $X$ if it satisfies the conditions (a),(b),(c) and $(\mathrm{d})$.

It obtains that if $\{S(t, s)\}_{t, s \geq 0}$ is a two-parameter integrated semigroup then both of the families $\{S(t, 0)\}_{t \geq 0}$ and $\{S(0, s)\}_{s \geq 0}$ are one-parameter integrated semigroups. Moreover from conditions (c) and (d) one can see that $S(t, s)$ commutes with $S\left(t^{\prime}, s^{\prime}\right)$, for all $t, t^{\prime}, s, s^{\prime} \geq 0$.

We say that the two-parameter integrated semigroup $\left\{S\left(t_{1}, t_{2}\right)\right\}_{t_{1}, t_{2} \geq 0}$ is exponentially bounded if there exist $M \geq 0$ and $\omega_{1}, \omega_{2} \in \mathbb{R}$ such that $\left\|S\left(t_{1}, t_{2}\right)\right\| \leq$ $M e^{\omega_{1} t_{1}+\omega_{2} t_{2}}$, for all $t_{1}, t_{2} \geq 0$ and we say that $\left\{S\left(t_{1}, t_{2}\right)\right\}_{t_{1}, t_{2} \geq 0}$ is non-degenerate if both one-parameter integrated semigroups $\left\{S\left(t_{1}, 0\right)\right\}_{t_{1} \geq 0}$ and $\left\{S\left(0, t_{2}\right)\right\}_{t_{2} \geq 0}$ are non-degenerate.

We define the ordered pair $(H, K)$ as the generator of $\{S(t, s)\}_{t, s \geq 0}$ if $H$ is the generator of the integrated semigroup $\{S(t, 0)\}_{t \geq 0}$ and $K$ is the generator of the integrated semigroup $\{S(0, s)\}_{s \geq 0}$. So we obtain $H, K$ as follows

$$
\begin{aligned}
& H=\lambda-R_{\lambda}^{-1} \text { in which } R_{\lambda}=\lambda \int_{0}^{\infty} e^{-\lambda t} S(t, 0) d t, \text { for } \lambda \in \rho(H) . \\
& K=\mu-R_{\mu}^{-1} \text { in which } R_{\mu}=\mu \int_{0}^{\infty} e^{-\mu s} S(0, s) d s, \text { for } \mu \in \rho(K) .
\end{aligned}
$$

Proposition 1.5 Let $H: D(H) \subseteq X \rightarrow X$ and $K: D(K) \subseteq X \rightarrow X$ be two densely-defined closed linear operators and there are $a, b \geq 0$ satisfying $(a, \infty) \subseteq \rho(H),(b, \infty) \subseteq \rho(K)$ and let $M \geq 0, \omega_{1} \in(-\infty, a]$ and $\omega_{2} \in(-\infty, b]$ then the following assertions are equivalent:

(i) $(H, K)$ generates a two-parameter integrated semigroup $\{S(t, s)\}_{t, s \geq 0}$ satisfying $\|S(t, s)\| \leq M e^{\omega_{1} t+\omega_{2} s}$, for all $t, s \geq 0$.

(ii) $\|\left(\lambda-\omega_{1}\right)^{k+1}\left[\frac{R(\lambda, H)}{\lambda}\right]^{(k)} / k$ ! $\| \leq M$, for all $\lambda>a, k=0,1,2, \ldots$.

$$
\begin{aligned}
& \left\|\left(\mu-\omega_{2}\right)^{k+1}\left[\frac{R(\mu, K)}{\mu}\right]^{(k)} / k !\right\| \leq M, \text { for all } \mu>b, k=0,1,2, \ldots . \\
& R(\lambda, H) R(\mu, K)=R(\mu, K) R(\lambda, H), \text { for all } \lambda>a, \mu>b .
\end{aligned}
$$

The first application of integrated semigroup was a result about resolvent positive operator that we explain some preliminaries about it here. One can 
see more details in [1].

Definition 1.6 A cone $C$ of vertex 0 is a subset of a vector space $L$ such that for each $x \in C$ and $\lambda>0, \lambda x \in C$. If in addition, $C$ is convex, then $C$ is called a convex cone of vertex 0 . Thus a convex cone of vertex 0 is a subset of $L$ such that

(i) $C+C \subset C$

(ii) $\lambda C \subset C$ for all $\lambda>0$.

A cone $C \subset L$ satisfying (i) and (ii) is said to be generating if $L=C-C$. Also if $C$ is a cone in topological vector space $L$ then the dual cone $C^{\prime}$ of $C$ is defined to be the set $\left\{f \in L^{\prime}: f(x) \geq 0\right.$ if $\left.x \in C\right\}$, where $L^{\prime}$ is the dual space of $L$.

A cone $C$ in a topological vector space $L$ is said to be normal if $\mathcal{U}=[\mathcal{U}]$, where $\mathcal{U}$ is a filter of neighborhoods of 0 . Hence $C$ is a normal cone if and only if there exists a base of C-saturated neighborhoods of 0 .

From now we assume that E denotes a real ordered Banach space whose positive cone $E_{+}$is generating and normal. For example, $E$ may be a Banach lattice or the hermitian part of a $C^{*}$-algebra.

Definition 1.7 An operator $A$ on $E$ is called resolvent positive if there exists $\omega \in \mathbb{R}$ such that $(\omega, \infty) \subset \rho(A)$ and $R(\lambda, A) \geq 0$ for all $\lambda>\omega$.

Now let $A$ be a resolvent positive operator. Define $s(A)=\inf \{\omega \in \mathbb{R}$ : $(\omega, \infty) \subset \rho(A)$ and $R(\lambda, A) \geq 0$ for all $\lambda>\omega\}, D(A)_{+}=E_{+} \cap D(A)$ and $D\left(A^{\prime}\right)_{+}=E_{+}^{\prime} \cap D\left(A^{\prime}\right)$, where in the second definition we assume that $A$ is densely defined and $A^{\prime}$ denotes the adjoint of $A$.

A subset $C$ of $E_{+}$is called cofinal in $E_{+}$if for every $a \in E_{+}$there exists $b \in C$ such that $a \leq b$.

If $\{T(t)\}_{t \geq 0}$ is a positive $C_{0}$-semigroup with generator $B$, then the type (or growth bound) $\omega(B)$ is defined by $\omega(B)=\inf \{W \in \mathbb{R}$ : there exists $M \geq 1$ such that $\|T(t)\| \leq M e^{W t}$ for all $\left.t \geq 0\right\}$. One always has $s(B) \leq \omega(B)<\infty$ (See [4] Chapter IV Proposition 2.2).

In the sequel, we state some conditions under which resolvent positive operator $A$ is the infinitesimal generator of a $C_{0}$-semigroup.

Theorem 1.8 Let $A$ be a densely defined resolvent positive operator on $E$. If $D(A)_{+}$is cofinal in $E_{+}$or if $D\left(A^{\prime}\right)_{+}$is cofinal in $E_{+}^{\prime}$, then $A$ is the generator of a positive $C_{0}$-semigroup. Moreover, $s(A)=\omega(A)$.

Proof. See [1] Theorem 2.2.

Corollary 1.9 Assume that int $E_{+} \neq \emptyset$. If $A$ is a densely defined resolvent positive operator on $E$, then $A$ is the generator of a positive $C_{0}$-semigroup and $s(A)=\omega(A)$.

Proof. See [1] Corollary 2.3.

Theorem 1.10 Let $A$ be a densely defined resolvent positive operator on $E$. If there exist $\lambda_{0}>s(A)$ and $c>0$ such that $\left\|R\left(\lambda_{0}, A\right) a\right\| \geq c\|a\|, a \in E_{+}$, 
then $A$ is the generator of a positive $C_{0}$-semigroup and $s(A)=\omega(A)$.

Proof. See [1] Theorem 2.5.

Theorem 1.11 Suppose that the norm is additive on the positive cone, that is $\|a+b\|=\|a\|+\|b\|$ for all $a, b \in E_{+}$(for example, $E=L^{1}(X, \mu)$ ). Let $A$ be a densely defined operator. The following assertions are equivalent:

(i) $A$ generates a positive $C_{0}$ - group (For this concept see [9]).

(ii) $A$ and $-A$ are resolvent positive and there exist $\lambda>\max \{s(A), \omega(A)\}$ and $c>0$ such that $\|R(\lambda, \pm A)\| \geq c\|a\|$ for all $a \in E_{+}$.

Proof. See [1] Theorem 2.6.

The following theorem shows that a densely defined resolvent positive operator $A$ is the infinitesimal generator of an increasing one-parameter integrated semigroup. This is based on the Hille-Yosida Theorem and can be applied when $A$ has a dense domain.

Theorem 1.12 Let $A$ be a densely defined resolvent positive operator. There exists a unique increasing integrated semigroup $\{S(t)\}_{t \geq 0}(S(t) \leq S(s)$ for $0 \leq t \leq s)$ of positive operators such that

$$
R(\lambda, A)=\int_{0}^{\infty} e^{-\lambda t} d S(t), \lambda>s(A) .
$$

Proof. See [1] Theorem 4.1.

In the pervious theorem it is not necessary to assume that $A$ has a dense domain if additional assumptions on the space are made.

Definition 1.13 We say that $E$ is an ideal in $E^{\prime \prime}$ if for $a \in E, b \in E^{\prime \prime}, 0 \leq$ $b \leq a$ implies that $b \in E$. Here $E$ is identified with a subspace of $E^{\prime \prime}$ (via evaluation) and $E_{+}^{\prime \prime} \cap E=E_{+}$(that is, $E$ is an ordered subspace of $E^{\prime \prime}$ ).

Theorem 1.14 Suppose that $E$ is an ideal in $E^{\prime \prime}$. Let $A$ be a resolvent positive operator. Then there exists a unique increasing integrated semigroup $\{S(t)\}_{t \geq 0}$ of operators on $E$ such that

$$
R(\lambda, A)=\int_{0}^{\infty} e^{-\lambda t} d S(t), \lambda>s(A) .
$$

Proof. See [1] Theorem 5.7.

By the result of the two preceding theorems, resolvent positive operator A generates a unique increasing integrated semigroup $\{S(t)\}_{t \geq 0}$ when either $A$ is densely defined or $E$ is an ideal in $E^{\prime \prime}$.

Note that the integrated semigroup generated by a resolvent positive operator is non-degenerate and increasing. The following theorem shows that conversely, every non-degenerate, increasing integrated semigroup is generated by a resolvent positive operator.

Theorem 1.15 Let $\{S(t)\}_{t} \geq 0$ be a non-degenerate, increasing integrated 
semigroup, then there exists a unique resolvent positive operator $A$ such that

$$
R(\lambda, A)=\int_{0}^{\infty} e^{-\lambda t} d S(t), \lambda>s(A)
$$

Moreover $D(A)$ is given by

$$
\begin{aligned}
& \{a \in E \text {; there exists a (necessarily unique) } b \in E \\
& \text { such that } \left.S(t) a=t a+\int_{0}^{t} S(r) b d r \text { for all } t \geq 0\right\}
\end{aligned}
$$

and so $A(a)=b$.

Proof. See [1] Theorem 6.8.

We terminate this section with the homogeneous abstract Cauchy problem associated to the resolvent positive operator.

Theorem 1.16 Assume that $A$ in (ACP)

$$
\left\{\begin{array}{l}
\frac{d u(t)}{d t}=A u(t) \quad t \in(0, T] \\
u(0)=x
\end{array}\right.
$$

is a resolvent positive operator and either $D(A)$ is dense or $E$ is an ideal in $E^{\prime \prime}$. For every $x \in D\left(A^{2}\right)$ there exists a unique solution of (ACP). Furthermore, denote by $\{S(t)\}_{t \geq 0}$ the integrated semigroup generated by $A$. Then $u(t)=\frac{\partial}{\partial t} S(t) x=A S(t) x+x$ for all $t \geq 0$. Moreover if $x \geq 0$ then $u(t) \geq 0$ for all $t \geq 0$.

Proof. See [1] Theorem 7.1.

\section{Resolvent Positive Operator and Two-Parameter Abstract Cauchy Problem}

We introduced resolvent positive operator and we stated some theorems about conditions that a resolvent positive operator generates a $C_{0}-$ semigroup. We can extend Theorem 1.8 for a pair of resolvent positive operators $\left(H_{1}, H_{2}\right)$. Throughout this section $E$ is a real ordered Banach space with generating and normal positive cone.

Theorem 2.1 Let $\left(H_{1}, H_{2}\right)$ be a pair of densely defined resolvent positive operators on $E$. If $D\left(H_{i}\right)_{+}$is cofinal in $E_{+}$or $D\left(H_{i}^{\prime}\right)_{+}$is cofinal in $E_{+}^{\prime}$, $i=1,2$ and also there exists $\omega \in \mathbb{R}$ such that $[\omega, \infty) \subseteq \bigcap_{i=1}^{2} \rho\left(H_{i}\right)$ and $R\left(\lambda, H_{1}\right) R\left(\lambda^{\prime}, H_{2}\right)=R\left(\lambda^{\prime}, H_{2}\right) R\left(\lambda, H_{1}\right)$ for all $\lambda, \lambda^{\prime} \geq \omega$, then $\left(H_{1}, H_{2}\right)$ is the generator of a positive $C_{0}$-two-parameter semigroup and $s\left(H_{i}\right)=\omega\left(H_{i}\right)$, $i=1,2$.

Proof. It is obvious by Hille-Yosida theorem for n-parameter semigroup and Theorem 1.8. $\square$

Similarly by using this additional condition (commutativity condition of 
resolvent operators) we can extend Corollary 1.9 and Theorems 1.10 and 1.11.

Also we can extend Theorems 1.12 and 1.14 for a pair of resolvent positive operators as follows

Theorem 2.2 Suppose that $H_{1}$ and $H_{2}$ are two densely defined operators or $E$ is an ideal in $E^{\prime \prime}$. If $H_{1}, H_{2}$ are resolvent positive operators and there exists $\omega \in$ $\mathbb{R}$ such that $(\omega, \infty) \subseteq \bigcap_{i=1}^{2} \rho\left(H_{i}\right)$ and $R\left(\lambda, H_{1}\right) R\left(\lambda^{\prime}, H_{2}\right)=R\left(\lambda^{\prime}, H_{2}\right) R\left(\lambda, H_{1}\right)$ for all $\lambda, \lambda^{\prime} \geq \omega$, then there exists a unique two-parameter integrated semigroup $\{S(t, s)\}_{t, s \geq 0}$ such that $\{S(t, 0)\}_{t \geq 0}$ and $\{S(0, s)\}_{s \geq 0}$ are increasing oneparameter integrated semigroups and

$$
R\left(\lambda, H_{1}\right)=\int_{0}^{\infty} e^{-\lambda t} d S(t, 0) \text { for } \lambda>s\left(H_{1}\right)
$$

and

$$
R\left(\lambda^{\prime}, H_{2}\right)=\int_{0}^{\infty} e^{-\lambda^{\prime} s} d S(0, s) \text { for all } \lambda^{\prime}>s\left(H_{2}\right) .
$$

Proof. By Theorems 1.12 and 1.14 there exist increasing one-parameter integrated semigroups $\left\{S_{t}\right\}_{t \geq 0}$ and $\left\{T_{s}\right\}_{s \geq 0}$ such that

$$
R\left(\lambda, H_{1}\right)=\int_{0}^{\infty} e^{-\lambda t} d S(t) \text { for } \lambda>s\left(H_{1}\right)
$$

and

$$
R\left(\lambda^{\prime}, H_{2}\right)=\int_{0}^{\infty} e^{-\lambda^{\prime} s} d T(s) \text { for } \lambda^{\prime}>s\left(H_{2}\right) .
$$

Now consider the family $S(t, s)=S_{t}+T_{s}$ for $s, t \geq 0$. From Proposition 1.5 it yields that $\{S(t, s)\}_{t, s \geq 0}$ is a two-parameter integrated semigroup (see [8] for more details).

Theorem 2.3 Let $\{S(t, s)\}_{t, s \geq 0}$ be a non-degenerate, two-parameter integrated semigroup such that both of one-parameter integrated semigroups $\{S(t, 0)\}_{t \geq 0}$ and $\{S(0, s)\}_{s \geq 0}$ are increasing. Then there exists a unique pair of resolvent positive operators $\left(H_{1}, H_{2}\right)$ such that

$$
R\left(\lambda, H_{1}\right)=\int_{0}^{\infty} e^{-\lambda t} d S(t, 0) \text { for } \lambda>s\left(H_{1}\right)
$$

and

$$
R\left(\lambda^{\prime}, H_{2}\right)=\int_{0}^{\infty} e^{-\lambda^{\prime} s} d S(0, s) \text { for } \lambda^{\prime}>s\left(H_{2}\right) .
$$

Moreover $D\left(H_{1}\right)$ is given by

$D\left(H_{1}\right)=\left\{a \in E: \exists ! b \in E\right.$ such that $S(t, 0) a=t a+\int_{0}^{t} S(r, 0) b d r$ for all $\left.t \geq 0\right\}$ 
where notation $\exists$ ! means "there exists a unique". In this case $H_{1}(a)=b$. Similarly $D\left(H_{2}\right)$ is defined by

$$
D\left(H_{2}\right)=\left\{a^{\prime} \in E: \exists ! b^{\prime} \in E\right.
$$

such that $S(0, s) a^{\prime}=s a^{\prime}+\int_{0}^{s} S(0, p) b^{\prime} d p$ for all $\left.s \geq 0\right\}$

and $H_{2}\left(a^{\prime}\right)=b^{\prime}$.

Proof. It is easily proved by Theorem 1.15 .

In the following theorem we discuss the two-parameter abstract Cauchy problem associated to resolvent positive operators.

Theorem 2.4 Consider 2-RACP

$$
\left\{\begin{array}{l}
\frac{\partial}{\partial t_{i}} u\left(t_{1}, t_{2}\right)=H_{i} u\left(t_{1}, t_{2}\right) \quad i=1,2, \quad t_{i} \in\left[0, a_{i}\right] \\
u(0,0)=u_{0}
\end{array}\right.
$$

Where $u$ is a function from $\left[0, a_{1}\right] \times\left[0, a_{2}\right]$ to ordered Banach space $E$ , $a_{1}, a_{2}>0, H_{i}: D\left(H_{i}\right) \subseteq E \rightarrow E, i=1,2$, is a resolvent positive operator and $u_{0} \in E$.

By a solution of 2-RACP we mean a continuous function $u:\left[0, a_{1}\right] \times\left[0, a_{2}\right] \rightarrow E$ in which $u$ has continuous partial derivatives and $u\left(t_{1}, t_{2}\right) \in \bigcap_{i=1}^{2} D\left(H_{i}\right)$ and $u$ satisfies in 2-RACP.

If either $H_{1}$ and $H_{2}$ are densely defined or $E$ is an ideal in $E^{\prime \prime}$ then for every $u_{0} \in \bigcap_{i=1}^{2} D\left(H_{i}^{2}\right)$ there exits a unique solution of the Cauchy problem 2-RACP. Furthermore, denote by $\{S(t, s)\}_{t, s \geq 0}$ the two-parameter integrated semigroup generated by $\left(H_{1}, H_{2}\right)$ then $u\left(t_{1}, t_{2}\right)=\frac{\partial}{\partial t_{1}} S\left(t_{1}, 0\right) \frac{\partial}{\partial t_{2}} S\left(0, t_{2}\right) u_{0}$ for all $t_{1}, t_{2} \geq 0$.

Moreover, if $u_{0} \geq 0$, then $u\left(t_{1}, t_{2}\right) \geq 0$ for all $t_{1}, t_{2} \geq 0$.

Proof. The proof of the existence and uniqueness of solutions is completely similar to the proof of Theorem 4.1 of [8] with the difference that $u_{0} \in$ $\bigcap_{i=1}^{2} D\left(H_{i}^{2}\right) \subseteq \bigcap_{i=1}^{2} D\left(\widetilde{H}_{i}\right)$. Also by Proposition 1.16 one can see that if $u_{0} \geq 0$, then $u\left(t_{1}, t_{2}\right) \geq 0$ for all $t_{1}, t_{2} \geq 0$.

\section{References:}

[1] W. Arendt, Resolvent positive operators, Proc. London Math. Soc. (3) 54 (1987), 321-349.

[2] W. Arendt, Vector-Valued Laplace Transforms and Cauchy Problems, Israel Journal of Mathematics Vol. 59, No.3, 1987, 327-352.

[3] P. L. Butzer and H. Berens, Semigroup of Operators and Approximation, Springer-Verlag New York (1967).

[4] K.J. Engle and R. Nagel, One-Parameter Semigroups for Linear Evolution 
Equations, Springer-Verlag, New York (2000).

[5] E. Hille and R. S. Phillips, Functional Analysis and Semigroups, Amer. Math. Soc. Colloq. Vol. 31, Providence R. I. (1957).

[6] M. Janfada and A. Niknam, On the n-Parameters Abstract Cauchy Problem, Bul. Aus. Math. Soc. Vol. 69(2004)[383-394].

[7] M. Janfada and A. Niknam, Two-Parameter Dynamical System and Applications, J. Science of I. R. Iran, to appear.

[8] M. Khanehgir ,M. Janfada and A. Niknam, Two-Parameter Integrated Semigroups and Two-Parameter Abstract Cauchy Problems, Jour. of Inst. of Math. and Comp. Sci.(Math. ser.) Vol. 18,No. 1 (2005)1-12.

[9] A. Pazy, Semigroups of Linear Operators and Applications to Partial Differential Equations, Appl. Math. Sci. Vol. 44, Springer-Verlag(1983).

Received: February 23, 2007 